Marquette University

e-Publications@Marquette

$6-2003$

\title{
Health Risks, Past Usage, and Intention to Use Weight Loss Products in Normal Weight Women with High and Low Body Dysphoria
}

B. L. Whisenhunt

Louisiana State University

D. A. Williamson

Louisiana State University

Richard G. Netemeyer

University of Virginia

J. Craig Andrews

Marquette University, craig.andrews@marquette.edu

Follow this and additional works at: https://epublications.marquette.edu/market_fac

Part of the Marketing Commons

\section{Recommended Citation}

Whisenhunt, B. L.; Williamson, D. A.; Netemeyer, Richard G.; and Andrews, J. Craig, "Health Risks, Past Usage, and Intention to Use Weight Loss Products in Normal Weight Women with High and Low Body Dysphoria" (2003). Marketing Faculty Research and Publications. 29.

https://epublications.marquette.edu/market_fac/29 
ORIGINAL RESEARCH PAPER

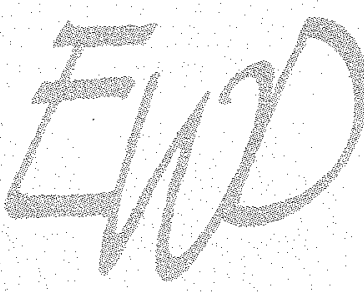

Health risks, past usage, and intention to use weight loss products in normal weight women with high and low body dysphoria

\author{
B.L. Whisenhunt*, D.A. Williamson*, R.G. Netemeyer**, and C. Andrew $S^{* * *}$
}

*Pennington Biomedical Research Center, Louisiana State University, Baton Rouge, LA, * Mcintire School of Commerce, Charlottesville, University of Virginia, VA, and **marquette University; Milwakee, WI, USA

\begin{abstract}
Objective: There are many health risks involved with the use of weight loss products by normal weight women. The mass media may compound this problem through the promotion of weight loss products and a thin body size. This study tested women's perceptions of different weight loss product ads to determine if body dysphoria (i.e., an over concern with body size and shape in normal weight people) was associated with risk beliefs, past behaviors, and intention toward using weight loss products. Method: Normal weight women (age range $=18-41 \mathrm{yr})$, who were classified as either high $(n=45)$ or low $(n=43)$ on a measure of body dysphoria, rated different weight loss products according to their perception of health risks, past behavior, and their intention to consume the products. These products were a dietary fat substitute (olestra), a prescription obesity medication (sibutramine), and an over-the-counter appetite suppressant (phenylpropanolamine). Results: High body dysphoric women reported higher intentions to use the products as well as increased prior use of two of the three weight loss products. High body dysphoric women did not believe that these weight loss products were harmless. They recognized potential health risks associated with using such products, but nonetheless, expressed intention to use these weight loss products at a higher frequency. Also, several variables related to body image were found to effectively discriminate normal weight women at risk for abusing weight loss products. Discussion: This study found that women who do not need to lose weight but have significant body image concerns were willing to use potentially harmful weight loss products despite the knowledge that such products might pose significant health risks. Techniques utilized by advertising regulatory agencies such as warning labels did not have a strong deterrent effect for stated intentions to use the products. Implications of these findings for public health policy issues were discussed.

(Eating Weight Disord. 8: 114-123, 2003). 2003, Editrice Kurtis
\end{abstract}

\section{:- INTRODUCTION}

Problems related to being overweight are estimated to account for 70 billion dollars annually in the United States in health-related costs (1). In addition, 33 billion dollars are spent per year on diet pills, exercise programs, and appetite suppressants, making dieting the norm rather than the exception (2). A recent report of a multi-agency conference on weight loss products and programs sponsored by the Federal Trade Commission (FTC), American Society for Clinical Nutrition, National Institute of Diabetes, and the Center for Disease Control and Prevention estimates that $40 \%$ of women in the U.S. are currently trying to lose weight (1). The primary reason for trying to lose weight is "a concern for physical appearance," and many of those attempting to lose weight are not overweight (based on height/weight ratios), i.e., normal weight women (1).

Ads promoting the use of weight loss products typically aim to promote a healthier lifestyle. However, these same ads may be interpreted in ways that are unhealthy by a minority of people who are "at risk" for psychological/physical problems (e.g., women with eating disorders). Several studies suggest that a motivating factor for unhealthy weight reduction behaviors may be media pressure in the form of weight loss ads. For example, Burton et al. (3) and 
Whisenhunt et al. (4) found a significant relationship between images in weight loss ads and unhealthy dieting behaviors. Rabak-Wagner et al. (5) reported that a primary factor affecting. unhealthy dieting in college-aged women is the information in ads for weight loss products. Grigg et al. (6) found that over half of the adolescent girls they sampled had used at least one unhealthy weight reduction method and $36 \%$. admitted to extreme weight loss methods including crash dieting, fasting, diet pills, and diuretic use under the assumption that these methods were harmless. In sum, normal weight women may choose to use weight loss products for aesthetic purposes under the misperception that such products are without health risk.

Women with body image disturbances may be at risk for abusing commercial weight loss products and may misinterpret the advertisements for these products. Research on cognitive biases has found that people diagnosed with an eating disorder tend to notice and recall information related to food and body: shape. Research on cognitive biases has also shown that normal weight women who are overly concerned with body size and shape (called body dysphoria) have information processing biases similar to women who meet the diagnostic criteria for an eating disorder (7). Cognitive biases are most prominent when ambiguous stimuli related to fatness, dieting, and control of food intake are presented. In a recent review, Williamson (8) reports that advertisements for weight loss products have many of the ambiguous features of the stimuli used in studies examining cognitive bias in body dysphoria.

Marketing research has suggested that consumers often misinterpret or misuse information presented in ads, particularly if the ad is ambiguous or does not include a disclosure $(9,10)$. For example, Burke et al. (11) found that consumers may misinterpret information presented in advertisements, but presenting truthful information/disclosures can serve to reduce such misinterpretations. Andrews et al. (9) examined how consumers interpret nutrient content claims made in advertisements. They found that the disclosures (in general) were successful in reducing favorable evaluations of disease-risk and nutrient content generalizations as compared to the no disclosure control group.

The current study tested how an "at risk" population (i.e., normal weight women with high body dysphoria) processes information in weight loss ads. The current study had two objectives: 1 to investigate the potential effects of advertisements for weight loss products in normal weight women high or low in body dysphoria, and 2) to identify body image variables that may put normal weight women at risk for abusing weight loss products, and that discriminate between high and low body dysphoric groups.

\section{METHOD}

Weight loss products studied

Three different classes of weight loss products were included in the study: a prescription obesity medication, a dietary fat substitute, and an over-the-counter appetite suppressant. Sibutramine the prescription drug selected for use in this study), is an appetite suppressant that works by inhibiting reuptake of norepinephrine, serotonin, and dopamine (12). Sibutramine can only be obtained through a physician's prescription, and it is only intended for use in people who have obesity which causes significant health problems that are greater than the potential side effects of the medication (increased heart rate and blood pressure). olestra (the dietary fat substitute selected for use in this study) is a fat substitute that gives food the same texture as fat but is not absorbed from the intestine and does not contribute to calories (3), One concern with this product is that some people may believe that olestra has a laxative effect, making it susceptible to abuse by persons with eating disturbances (14). The over-the-counter appetite suppressant selected for use in this study contains phenylpropanolamine (PPA), a sympathomimetic amine used to curb the appetite (15). 1 Each of the three weight loss products contains a label on the package describing possible side effects.

\section{Participants}

A total of 626 females enrolled at a major university were screened for inclusion in the study sample. To be included in the study, participants were required to 1) have high or low body dys phoria as defined by their score on the Body Shape Questionnaire (BSQ) (16), 2) be in the nor mal body weight range, as defined by a body mass index (BMT $=\mathrm{kg} / \mathrm{m}^{2}$ ) between 20 and 25 , and 3) be older than 18 years of age. Participants considered high in body dysphoria obtained a score in the upper quartile on the BSQ, while partici pants considered low in body dysphoria obtained

1Following data collection in this study, phenylpropanolamine was taken of the market by the Food and Drug Administration due to concerns about health risks associated with the use of this medication. 
a score in the lower quartile on the BSQ. Based on data from Muller et al. (17), cut-off scores on the BSQ for the upper and lower quartiles were established (upper quartile $\geq 118$; lower quartile $\leq 67 ; \mathrm{M}=93.99, \mathrm{SD}=35.02$ ).

Of the 626 initially screened, 105 women (age range $=(8-41)$ participated in the second phase of the study based on their classification as either high or low body dysphoric and their self-reported normal weight. Participants were tested 1 day to 1 week after the initial screening. The BSQ was re-administered at the time of testing to ensure that the test scores remained in the upper or lower quartiles. Participants who obtained BSQ scores within 5 points of the original cut-off scores were included in the study (resulting in new cut-off scores of 113 and 72 for high and low body dysphoria respectively). Those who scored greater than 5 points above or below the cut-off score on the second administration of the BSQ were excluded from the study. Participants who had an eating disorder as defined by DSMIV or were not of normal weight were excluded from the study. In the second phase of the study, 17 of the 105 participants were excluded from the study because: a) BSQ scores were 5 points above or below the cut-off $(n=2)$; b) BMI was below 20 or greater than $25(n=12)$; or $c)$ the presence of an eating disorder $(n=3)$. The final sample consisted of 88 women, 45 with high body dysphoria and 43 with low body dysphoria. The mean weight of participants was 130 pounds $(\mathrm{SD}=12.0 \mathrm{lbs})$, and mean height was 64 inches ( $\mathrm{SD}=2.3$ inches). The mean age of participants did not differ across the high $(\mathrm{M}=19.4, \mathrm{SD}=2.0)$ and low $(\mathrm{M}=20.6, \mathrm{SD}=4.0)$ body dysphoria groups. This sample of women included a diverse mixture of racial and ethnic groups: $76 \%$ Caucasian, 13\% African American, 6\% Asian, $2.5 \%$ Hispanic, and $2.5 \%$ Native American.

\section{Procedure}

In the first phase of the study, participants were screened using the BSQ and self-reported height and weight. Participants who met the primary inclusion criteria for the study were asked to return. In the second phase of the study, participants were told that the experiment was designed to assess consumer's attitudes about various products based on viewing advertisements for those products. After viewing the ads, participants responded to the dependent measures and a memory quiz included to test the participants' comprehension of the information presented in the ads. Next, participants completed a questionnaire packet containing all other measures, were weighed and measured, and screened for eating disorder symptoms.

\section{Measures: Classification/screening variables}

Body Shape Questionnaire (BSQ)

To examine our first study objective, a measure of body dysphoria was needed to classify subjects as high or low in body dysphoria. As previously stated, the BSQ was used. The BSQ is a 34 -item self-report questionnaire used to assess concerns about body shape and size where all items are scored on 6-point Likert scales ranging from "never". to "always." The term body dysphoria was coined by Williamson et al. (18) to describe normal weight people who are preoccupied with body size and shape (but not other body concerns). Williamson hypothesized that this group of nonclinical subjects would have many of the characteristics of people diagnosed with eating disorders. In subsequent studies, the BSQ has proven to be useful in defining nonclinical subjects of normal weight status with weight concerns similar to eating disordered patients (19).

Interview for the diagnosis of eating disorders - Fourth revision (IDED-IV)

The IDED-IV is a semi-structured clinical interview found to be a valid and reliable assessment instrument for diagnosing anorexia nervosa, bulimia nervosa, binge eating disorder, and eating disorder not otherwise specified based on DSM-IV criteria (20). The IDEDIV was administered to participants as a screening tool for the presence of an eating disorder. Three participants were excluded from participation based upon this screening interview.

\section{Measures: Independent/discriminator variables}

To examine our second objective, several body-image related independent variables were measured and used for discriminant analyses. All of these measures were responded to in the second phase of the study, after the experiment was conducted. These measures are as follows:

Three-Factor Eating Ouestionnaire (TFEO) Restrictive eating attitudes were measured with the three scales of the TFEQ: 1) dietary restraint, 2) disinhibition, and 3) perceived hunger. All three scales have been reported to have good test-retest reliability (21). The TFEQ was used to examine whether differing levels of eating restraint are associated with high ratings of intent to use weight loss products, and if the TFEQ scales were effective discriminators of high and low body dysphoric groups. 
Psychosocial Risk Factors Questionnaire (PRFO)

The PRFQ is an 18-item self-report measure containing four sub-scales: 1) concern for physical appearance, 2) media pressure for thinness, 3) social pressure for thinness, and 4) perception of physical appearance. The PRFQ has demonstrated adequate concurrent validity and test-retest reliability for each of the four subscales (4). The PRFQ scales are used to examine whether its sub-scales are associated with high ratings of intent to use weight loss products, and if they were effective discriminators of high and low body dysphoric groups.

\section{Beck Depression Inventory - Second edition} (BDI-II)

The BDI-II is a 21-item self-report measure designed to assess severity of depressive symptoms (22). The BDI-II was used to determine if it was associated with high ratings of intent to use weight loss products, and if it was an effective discriminator of high and low body dysphoric groups.

\section{Measures: Dependent variables}

\section{Past Product Use}

Past usage of diet products was assessed via the following question for each of the three products: Have you ever used the product or one similar to it? (yes/no).

\section{Intention and risk}

For each product, participants completed seven items adapted from Andrews et al. (9, 23) assessing behavioral intention to use the product in the future and the perceived risk

TABLE 1

Disclosures for the three weight loss products.

Control version

When colling
lo ploce
your order,
mention his
doverisenent

\section{Product \\ PPA Disclosure version

PPA plls
moy couse
yyperension,
selzures, or psydholic readions}

This product may cause abdominal cramping, noused ordidrheo

look for
moneysang
coupons in
yourlocal
newspaper

Sibutromine

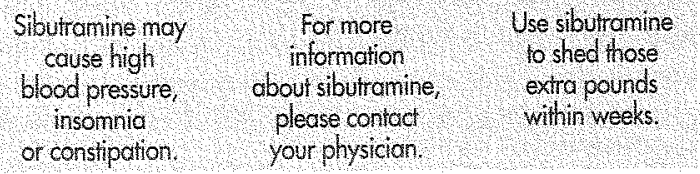

associated with the product. This division was based on theoretical grounds as well as empirical evidence. The two questions comprising the behavioral intention scale ("How. likely would you be to use this product in the future?" and "How often would you consider using this product?") were highly correlated $(\mathrm{r}=0.71, \mathrm{p}<0.001)$ but were not highly correlated with the other three items. The remaining three questions comprised the perceived risk scale ("Do you consider this product to be healthy/unhealthy for you?" " Do you agree/disagree that regular use of this product would contribute to the risk of health problems?" and "Do you believe that the benefits gained from using this product regularly would outweigh the risks involved?") and were also positively correlated with $r$-values ranging from 0.33 to 0.55 ) (Coefficient alpha $=$ $0.67)$. Each question could be rated from 0 to 4. Therefore, by adding the ratings, the range of possible scores for the behavioral intention scale was 0 to 8 , and the range of possible scores for the perceived risk scale was 0 to 12 .

\section{Advertising stimuli}

The advertising stimuli consisted of three experimental ads of weight loss products and six dummy ads. The six dummy ads were included as test stimuli in order to disguise the purpose of the study and included the following products:

1) over-the-counter headache medication containing acetaminophines, aspirin and caffeine, 2) prescription contraceptive, medroxyprogesterone, 3) tampons, 4) prescription allergy medication, fexofenadine hydrochloride, 5) hair dye and 6) whitening toothpaste. Original advertisements in published journals or magazines were used. As previously stated, the experimental products were olestra, sibutramine, and PPA. Each product had three versions (nine ads total): one containing additional information about the health risks associated with the product (deterrence version), one containing additional information unrelated to the product's function (control version), and one containing additional information intended to enhance perceptions of the product's efficacy (enhancement version). The information added to each of the weight loss ads according to disclosure version is shown in Table 1. All of the ads were in full color. For each version of all nine ads (three experimental and six dummy ads), additional information was added at the bottom of the ad in a white box, keeping font size and number of additional words (nine words) constant across all ads. Within the entire study, each ad had three versions of product disclosure statements, but any one participant viewed only one version of each 
ad with the same type of product disclosure statement (deterrent, control, or enhancement) for all of the weight loss ads.

Prior to initiation of the study, two steps were taken in order to establish the content validity of the advertising stimuli. First, two expert judges, each with over 15 years of research experience in marketing and advertising research, rated the advertisements. The raters were asked to examine each of the three versions of the weight loss product advertisements and rate them according to several dimensions. Expert ratings indicated high levels of authenticity, salience, and consistency with actual advertising formats (mean ratings ranging from 5.0 to 6.3 on a 7 -point Likert type scale). The second step taken to ensure the content validity of the ad stimuli was a pilot test of the advertisements with college student participants. Participants included 30 undergraduate females who viewed all nine advertisements. After viewing each of the nine ads, participants completed the dependent variable measures and a four-item memory quiz concerning the content of the advertisements. The quizzes were included as a manipulation check to determine how well participants attended to the information presented in the ads as well as to test the possibility of fatigue effects. Participants were later asked open-ended questions about what they thought the study was about, whether or not they believed that there was extra information added to the ads, whether or not they noticed anything strange about the ads, or if they thought the ads appeared realistic. There was no order effect for the quiz scores indicating the absence of a fatigue effect, and none of the participants accurately guessed the purpose of the study.

\section{RESULTS}

\section{Objective one: Comparisons for past} product usage, intention, and risk

We hypothesized that women with high levels of body dysphoria would report higher past usage of the weight loss products than would women with low levels of body dysphoria. Table 2 summarizes percentages of positive and negative responses across the three different products as a function of level of body dysphoria. A 2X2 chi-square contingency table indicated that responses to the question "Have you ever used the product or one similar to it?" differed significantly as a function of body dysphoria for PPA $\left[\mathrm{X}^{2}(1)=15.10, \mathrm{p}<0.0001\right]$ and sibutramine $\left[\mathrm{X}^{2}(1)\right.$ $=4.74, p<0.05]$. That is, for PPA and sibu-
TABLE 2

Prior knowledge and/or use of the experimentl weight loss products.

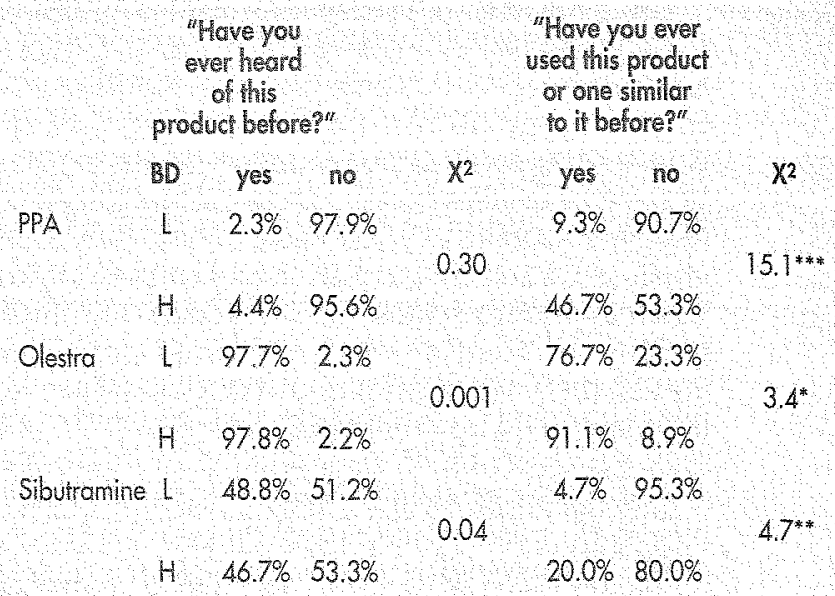

Note: BD = body dysphoria group, $\mathrm{L}=$ low, $\mathrm{H}$ thigh

$* 0<0.10, * 0<0.05^{* * *} 0<0.01$

tramine, participants with high levels of body dysphoria reported increased prior use of the product (or a similar product) as compared to participants with low levels of body dysphoria. There was only a marginally significant result for olestra $\left[\mathrm{X}^{2}(1)=3.40, \mathrm{p}<0.10\right]$. (As a data check, responses to the question "Have you ever heard of this product before?" did not differ across high and low body dysphoric groups for any of the three weight loss products) (Table 2). Thus, the hypotheses that past usage would be higher for the high dysphoric group was supported for two products, and was marginally supported for the third.

We also hypothesized that women with high levels of body dysphoria would report a higher intent to purchase and a lower perceived risk for the weight loss products than would women with low levels of body dysphoria. Multivariate Analysis of variance (MANOVA) was used to evaluate group differences in ratings on intention and risk. A 2 (level of body dysphoria) $x 3$ (ad version) x 3 (product type) repeated measures MANOVA was used. The MANOVA was performed with two between subjects factors (level of body dysphoria and ad version) and one within subjects factor (product type).2 Significant main effects were obtained for level of body dysphoria $[F(2,81)=19.80, p<0.0001]$, product type $[F(4,79)=67.61, p<0.0001]$, and ad version $[F(4$, $164)=2.43, p=0.05]$. There were no significant interactions between body dysphoria, ad version, or product type. 
TABLE 3

Means and standard deviations on the behavioral intentions scale and perceived risks scale for the three weight loss products.

\section{Product}

PPA

48

olestro

Clesira siburramine
Sin

\begin{abstract}
Product altitudes
\end{abstract} questionnaire scale

Behavioral intertions

Perceived risks erceived risks

Behovioral intentions

Perceived risks

Behavioral intentions

Perceived risks
Behoviord inientions

Porceived risk

$$
\text { Level of bo }
$$$$
\begin{aligned}
& \text { Low } \\
& \text { High }
\end{aligned}
$$$$
\text { Low }
$$$$
\text { High }
$$

Low

High

Low

High

Low

Low

High

\section{y dysphorio} ow

Tosal

Totd

Told

$$
4
$$

Total

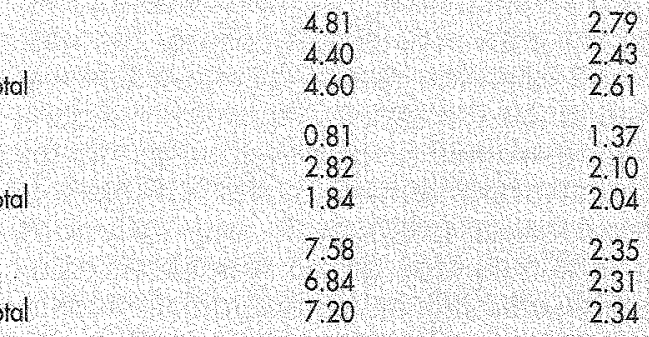

Analyses of variance (ANOVA) for each dependent variable were conducted as followup tests to the MANOVA. The ANOVA for main effect of level of body dysphoria was significant for the behavioral intention scale [F (1, $82)=32.31, p<0.0001]$, but not for the perceived risk scale $[F(1,82)=3.13, p>0.05]$. High body dysphoria was associated with higher intent to use PPA $[F(1,82)=17.75, p<0.0001]$, olestra $[F$ $(1,82)=12.84, p<0.05]$, and sibutramine $[F(1$, $82)=27.71, p<0.0001]$. There was no main effect of body dysphoria for perceived risk associated with PPA $[F(1,82)=1.86, p>0.05]$, olestra $[(F(1$, $82]=0.51, p>0.05]$, or sibutramine $[F(1,82)=$ $2.18, p>0.05]$. Table 3 summarizes the means and standard deviations for each of the three products on the behavioral intention scale and perceived risk scale.

A second hypothesis was that a "deterrent disclosure" (i.e., negative health side effects) would result in the lowest behavioral intention score and highest risk score in comparison to a "control" (neutral information) or "enhancement disclosure" (i.e., positive weight-loss effects of the product). The only significant effect for ad version was found for olestra $[\mathrm{F}(2,82)=4.48, \mathrm{p}<0.05]$. Tukey's HSD post hoc analyses were conducted for the main effect of ad version on the olestra intention scale to determine which ad condition affected olestra behavioral intention scores most strongly. The olestra intention score for the deterrence disclosure $(M=3.23, S D=2.18)$ was significantly lower than the control $(\mathrm{M}=4.34, \mathrm{SD}=1.59)$ and enhancement ( $M=4.45, S D=1.48)$ ad versions. The olestra intention scores for the control and enhancement product disclosure statements did not differ significantly. All interaction effects involving ad version were nonsignficant ( $p>0.05$ ). These findings indicate that the risk disclosure reduced behavioral intention to use olestra, but did not significantly influence intent to use the other weight loss products.

Univariate analyses also found significant differences among the behavioral intention [F $(2,164)=92.9, p<0.0001]$ and perceived risk $[F$ $(2,164)=103.96, p<0.0001]$ scores for different product types. Bonferroni adjusted pairwise comparisons indicated that both high and low body dysphoric groups rated olestra with significantly higher intentions to use and lower in perceived risk than sibutramine and PPA. Sibutramine, a prescription medication, was rated with higher behavioral intention to use and lower in perceived risk than was PPA, an overthe-counter medication.

\section{Objective two: Discriminators of high intention, and high and low body dysphoria}

A third hypothesis was that women with the highest levels of intention to use the weight loss products would have higher scores on measures of restrictive eating, concern for and perception of physical appearance, social and media pressure for thimness, and depression when compared to women with lower intention to use the three weight loss products. To test 
this hypothesis, discriminant function analysis was conducted. This analysis tested whether eight variables (PRFQ: concern for physical appearance, media pressure for thinness, perception of physical appearance, social pressure for thinness, BDI-II, TFEQ-restraint, disinhibition, and perceived hunger) differentiated women who obtained high scores on the behavioral intention scale for the three weight loss products. Women were defined as having high behavioral intention to use the products if they obtained a behavioral intention score 1.5 SD or more above the mean for 2 out of the 3 weight loss products, PPA and sibutramine. (Given the non-significant results for past usage and intention results for olestra, it was not used in these analyses.) According to these criteria, 12 women were classified as having high behavioral intention scores. Of these 12 women, 11 were in the high body dysphoria group. A comparison group was obtained by randomly selecting an additional 12 women from the sample. The overall Wilks' Lambda was significant, $\Lambda=0.29, \chi^{2}(8)=19.67, p=0.012$, indicating that the two groups differed on their psychological profiles. The top of Table 4 presents the standardized canonical discriminant function coefficients as well as group means and SD. The discriminant analysis found that $91 \%$ of the individuals in the sample were correctly classified. Further, with the exception of TFEQ-H, the means for the high intention group were greater than the means for the low intention group across variables (F-values $\geq 14.21 ; p<0.01$ ).

A fourth hypothesis was that the high body dysphoria group would have higher scores on measures of restrictive eating, concern for and perception of physical appearance, social and media pressure for thinness, and depression when compared to the low body dysphoria group. To test this hypothesis, discriminant function analysis was conducted to determine if the same eight variables differentiated women previously classified as high $(n=45)$ and low in body dysphoria $(n=43)$. The overall Wilks' Lambda was significant, $\Lambda=0.20, \chi^{2}(8)=123.83$, $p<0.01$, indicating that the two groups differed on their psychological profiles. The bottom of Table 4 presents the standardized canonical discriminant function coefficients, group means, and SD. The discriminant analysis found that $100 \%$ of the individuals in the sample were correctly classified, and with the exception of TFEQ- $H$, the means for the high dysphoric group were greater than the means for the low dysphoric group across variables (F-values $\geq 29.46 ; p<0.01$ ).

The results suggested that women who had strong intentions to use these weight loss products exhibited higher dietary restraint, higher perceived social pressure for thinness, lower perceived physical attractiveness, higher concern for physical appearance, higher perceived media pressure for thinness, higher susceptibility for overeating, and higher levels of depression. Thus, the participants who expressed strong intentions to use these weight loss prod-

TABLE 4

Hypothesis 3: Siandardized coefficients and predictor variables with the discriminant function.

Standerdized
cononical Mean (SD)
coefficients
With discriminaty
Tunchion

\begin{tabular}{|c|c|c|c|}
\hline & & & \\
\hline Th & 0.44 & $13.10(4.18)$ & $7.27(4.73)^{*}$ \\
\hline PRPQSPT & 0.42 & $25.36(2.91)$ & $19.27(6.20)$ \\
\hline PRPQPPA & 0.42 & $14.64(5.14)$ & $2118(5.34)^{*}$ \\
\hline PRFQ-CPA & 0.40 & $29.54(4.46)$ & $22.18(7.52)^{*}$ \\
\hline PRFQ-MPT & 0.37 & $24.09[4.23)$ & $1791(6.64)^{\circ}$ \\
\hline TFEQ-D & 0.36 & $10.36(4.18)$ & $6.45(4.46)^{6}$ \\
\hline BDIII & 0.35 & $19.82(8.44)$ & $10.27(9.80)^{*}$ \\
\hline THEOH & -0.07 & $7.09(3.96)$ & $782(3.54)$ \\
\hline
\end{tabular}

Hypothesis 4. Standardized coefficients and prediclor variables with the discriminant function

Sondordized
cononical
coeficients
with discrininght
Wunction

Mewn (SD) Low oysphorio function

Varable

\begin{tabular}{|c|c|c|c|}
\hline THEOR & 0.51 & $1786(4,42)$ & $4.05(3.2)^{\circ}$ \\
\hline PRP SPT & 0.25 & $2431(2.83)$ & $1708\left(5.10^{\circ}\right.$ \\
\hline PRFO PPA & 0.36 & $14.69(1.60)$ & $21.03 / 5,12)^{\circ}$ \\
\hline PRFOCPA & 0.04 & $27.29635)$ & $20.43(6.08)$ \\
\hline PRFQ $\mathrm{MPT}$ & 042 & $23,2(3.05)$ & $14.43(4,25)$ \\
\hline THEO & 027 & $1002(3,23)$ & $4.30(3.84)$ \\
\hline 111 & 033 & $18.71(8.62)$ & $5.10(4.49)^{\circ}$ \\
\hline $\mathrm{QH}$ & 0.03 & 90013541 & $675(8.52)$ \\
\hline
\end{tabular}

Note: TFEO R - Three-factor Ealing Questionnaire Restrain Scale, PRFO-SPT = Psychosocial Risk foctors Questionnaire Socid Pressure Cor Thimess Scde, PRFQ. PPA = Psychosocid Rist Factors Questionnaire Perception of Ply sical Atroctiveness Scale, PRFO CPA = Pychosocid Risk Fociors Questionnaire Concern for Physicd Appearance Scole: PRFO UPT - Psychosocid Risk factors Questionnaire Medic Pressure for Thinness Scale; TFEOD - Three-Focior Eaing Questiomaire Disinhibition Scale, BDIII = Beck Depression Inventory-Second Edition, TFE-H - Three-Facior Eoting Questionnaire Perceived Hunger Scale Differences between groups are signicont at the 0.01 level. 
ucts displayed many of the characteristics of persons diagnosed with an eating disorder but did not report the severity of symptoms that are commonly associated with eating disorders. Similarly, high body dysphoric women scored higher than low body dysphoric women on the same seven body image variables.

\section{DISCUSSION}

Before discussing the findings of the study, it is important to recognize some of the limitations of the study. The participants were young American women and the results should not be generalized to other cultures since many ethnic groups were not represented and the sample size was too small to allow for analyses of different ethnic groups. Also, the findings are based purely upon self-report of the participants. Therefore, we cannot conclude that these participants did in fact use the weight loss products simply because they reported an intention to do so. The results should be interpreted in the context of these considerations.

This study tested how normal weight women with body dysphoria responded to information in weight loss ads. In addition, this study examined select body image variables that contribute to an "at risk" group's intention to abuse weight loss products, and that discriminate between high and low body dysphoric groups. The hypothesis that normal weight women with high body dysphoria would have higher past usage and intention to use weight reduction products than would women with low levels of body dysphoria was largely supported. Specifically, high body dysphoric women reported higher intention to use the products in the future as well as actual higher prior use of two of the three weight loss products. However, women with high and low body dysphoria did not differ in their perceived risks for using the products, and in general, participants rated the weight loss products as having some health risks. (The overall means for risk, on a 0 to 12 scale, were 9.36 for PPA; 7.2 for sibutramine; and 4.6 for olestra.) This pattern of findings suggests that normal weight women with high levels of body dysphoria did not believe that these weight loss products were harmless. They recognized potential health risks associated with using such products, but nonetheless, expressed stronger intention to use these weight loss products.

In the context of research on cognitive biases associated with body dysphoria, these findings suggest that information processing with these ads was not completely unbiased. It is likely that high body dysphoric women had preexisting (and more favorable) attitudes about weight-loss products. Research on cognitive biases has postulated that strongly held beliefs (schemas) influence the processing of food and diet related information, resulting in unhealthy behavior to control body weight and shape (7). Our findings are consistent with this view.

The hypothesis that risk and intention would be affected by type of disclosure was not supported for two of three products. (Only olestra showed an effect). This finding is not consistent with the findings of previous studies $(11,24)$. One possible explanation might be that the ratings for intent to use PPA and sibutramine were generally low across all participants (i.e, 1.38 for PPA and 1.84 for sibutramine on a scale of 0 to 8 ). Thus, a "floor" effect may have been operating making it difficult to detect the impact of deterrent disclosures. In comparison, the olestra ratings were much higher than the PPA or sibutramine ratings, which may have allowed the finding of a suppressive effect for the deterrent risk disclosure. These results are consistent, however, with research demonstrating that similar disclosures can have different effects across product categories that are viewed differently in terms of initial attitudes and risk (cf. 9 vs 23). There was no effect of ad disclosure on perceived risk for any of the products. Even when risk factor information was added to the ads, participants did not rate the product as having more potential health risks, suggesting that women may have prior beliefs about the relative risks of certain products that are unchanged by information presented in the ads. This finding may have implications for future advertisement regulations, demonstrating the relative ineffectiveness of warning labels and health risk disclosures for certain types of products. Women with high body dysphoria indicated a general understanding of the risks involved with using the weight loss products regardless of which type of ad disclosure they received; however, knowledge of such health risks does not appear to be a deterrent for future use of such products. Perhaps reading about possible side effects is not an effective deterrent because it is not personally salient to the person. Future research should focus on developing effective methods for deter ring the inappropriate use of products that are potentially harmful.

Though hypotheses were not formally advanced with regard to product type, participants in both groups exhibited a similar pattern of responding, Olestra was the most highly rated product (in terms of lowest risk and highest intention) followed by sibutramine and PPA. It is not surprising that olestra was the most 
highly rated given that potato chips containing olestra are an easily accessible product, even though the olestra ingredient has been criticized by the Center for Science in the Public Interest for absorption and nutrient deprivation problems (25). However, the fact that ratings for sibutramine, a prescription medication, were more favorable than the ratings for PPA, an over-the-counter medication, was unexpected. A possible explanation for this is that people may be more trusting of medications that must be prescribed by a physician, thus affecting risk and intention. Thus, it is possible that prescription medications may be at higher risk for abuse compared to OTC medications. A significant number of participants reported prior use of the weight loss products despite their normal weight status. It was especially alarming that eleven women ( $20 \%$ of the high body dysphoria group) reported prior use of a prescription obesity medication even though they were of normal weight. These products are explicitly not recommended for people who are not clinically obese because of the risk of side effects associated with use of the medications. Additionally, a large proportion of the women in the high body dysphoria group (47\%) reported prior use of PPA or other OTC appetite suppressants. It is possible that the women who reported prior use of a prescription obesity medication or OTC appetite suppressant were obese while using the product, and lost enough weight to fall within a normal weight range and be eligible for participation in this study. However, given the serious side effect profile of these medications, future studies should address the question of the attainability of these medications and what measures could be taken to monitor improper use of such medications.

The results of the discriminant analyses show that a subset of normal weight women rated their intentions to use weight loss products significantly higher than the rest of the sample. Women at risk for inappropriate use of these products tended to actively restrain their eating, feel high pressure for thinness, and have higher levels of depression. Further, these same variables discriminated high and low body dysphoric groups. These results suggest that it is important for healthcare professionals and regulatory agencies to effectively identify women who are normal weight that are motivated to use potentially harmful weight loss products. Thus, mental health professionals as well as physicians may be able to identify those women who are willing to use extreme measures to attain weight loss by using simple self-report measures such as those utilized in the present study.

In summary, the results of this study suggest that there is cause for concern regarding potential misuse of weight loss products. Advertisements featuring the products used in this study as well as countless others are growing in number. Women who do not need to lose weight but have significant body image concerns appear to be willing to use potentially harmful weight loss products despite the knowledge that such products may pose significant health risks. Techniques utilized by advertising regulatory agencies such as warning labels do not appear to have a strong deterrent effect with normal weight women who are enrolled in college. Future research in this area should focus on developing ways to prevent the misuse of weight loss products through advertising, medical and mental health screenings, and improved product labels. For example, more creative policy measures may be needed, such as the use of prominent "black box" warnings employed for "fen-phen" (26), and other visual cues for conveying risk information. Such disclosures may help at-risk women make more informed decisions. However, given that our results show that women high in body dysphoria understand the risks, but largely choose to ignore such risks, stronger control of such products may be needed. For example, it has been suggested that many doctors and clinics have attracted "at-risk" populations via ads for products that claim safe weight loss. Our results pertaining to sibutramine and related prescription weight loss products are consistent with this suggestion. It may be that federal agencies, such as the FDA, FTC, and CDC, need to become more involved in the dissemination of risk information and in the regulation of the health care professionals' role in the promotion of weight loss products.

\section{REFERENCES}

1. Cleland R., Graybill D.C., Van Hubbard L.K.K., Stern J.S., Wadden T.A., Weinsier R., Yanovski S.: Commercial weight loss products and programs: What consumers stand to gain and lose a public conference on the information consumers need to evaluate weight loss products and programs. Clin. Rev. Food Sci. Nutr., 41, 45-70, 2001.

2. Berzins L.G.: Protecting the consumer through truth-in dieting laws. J. Soc. Issues, $2,371-382$, 1999.

3. Burton S., Netemever R.G., Lichtenstein D.: Gender differences for appearance-related attitudes and behaviors: Implications for consumer welfare. J. Public Policy \& Marketing, 13, 60-75, 1995.

4. Whisenhunt B.L., Williamson D.A., Netemeyer 
R.G., Womble L.G.: Reliability and validity of the psychosocial risk factors questionnaire (PRFQ). Eat. Weight Disord, 5, 1-6, 2000.

5. Rabak-Wagner J., Eickhoff-Shemek J., KellyVance $L$ : The effect of media analysis on attitudes and behaviors regarding body image among college students. J. Am. Health College, 47, 29-35, 1998.

6. Grigg M., Bowman J., Redman S., Disordered eating and unhealthy weight reduction practices among adolescent females. Prev. Med., 25, 748756, 1996.

7. Williamson D.A., Muller S.M., Reas D.L., Thaw J.A.: Cognitive bias in eating disorders: Implications for theory and treatment. Behav. Modif., 23, 556-577, 1999.

8. Williamson D.A.: Body image disturbances: A form of cognitive bias? Eating Disorders, 4, 4758, 1996.

9. Andrews J.C., Netemeyer R.G., Burton S.: Consumer generalization of nutrient claims on advertising. J. Marketing, 62, 62-75, 1998.

10. Hoch S.J., Young-Won Ha: Consumer learning: Advertising and the ambiguity of product experience. J. Consumer Res., 13, 221-233, 1986.

11. Burke R.R., DeSarbo R.L.O, Robertson T.S.: Deception by implication: An experimental investigation. J. Consumer Res., 14, 483-494, 1988.

12. Luque C.A., Rey J.A.: Sibutramine: A serotoninnorepinephrine reuptake-inhibitor for the treatment of obesity. Ann. Pharmacother., 33, 968$978,1999$.

13. Bergholz C.M.: Safety evaluation of olestra, a nonabsorbed, fat-like fat replacement. Crit. Rev. Food Sci. Nutr., 32, 141-146, 1992.

14. Hampl J.S., Sheeley A.E., Schnepf M.I.: Sounding the alarm for misuse of olestra-containing foods in binge-eating disorders [Letter to the editor]. J. Am. Diet. Assoc., 98, 971, 1998.

15. Bulik C.M.: Abuse of drugs associated with eating disorders. J. Subst. Abuse, 4, 69-90, 1992.

16. Cooper P. J., Taylor M.J., Cooper Z., Fairburn C.G.: The development and validation of the
Body Shape Questionnaire. Int. J. Eat. Disord, 6, 485-494, 1987.

17. Muller S.M., Williamson D.A., Martin C.K. Reconstructing reality: The false consensus bias and its relationship to body dysphoria. Poster presented at the $32^{\text {nd }}$ annual convention of the association for the advancement of behavior therapy, Washington, DC, 1998.

18. Williamson D.A., Barker S.E., Bertman L.J., Gleaves D.H.: Body image, body dysphoria, and dietary restraint: Factor structure in nonclinical subjects. Behav. Res. Ther, 33, 85-93, 1995.

19. Jackman L. J., Williamson D.A., Netemeyer R.G., Anderson D.A.: Do weight preoccupied women misinterpret ambiguous stimuli related to body size? Cognitive Ther. Res., 19, 341-355, 1995.

20. Kutlesic V., Williamson D.A., Gleaves D.A., Barbin J.M. Murphy-Eberenz K.P.: The Interview for the Diagnosis of Eating Disorders IV: Application to DSM-IV diagnostic criteria. Psychol. Assess., 10, $41-48,1998$.

21. Stunkard A.J., Messick S. The Three-Factor Eating Questionnaire to measure dietary restraint, disinhibition, and hunger. J. Psychosom. Res., 29, 71-83, 1985.

22. Beck A.T, Steer R.A., Brown G.K. Beck Depression Inventory-2 ${ }^{\text {nd }}$ Edition. The Psychological Corporation. San Antonio, Harcourt Brace \& Company, 1987.

23. Andrews J.C., Burton S., Netemeyer R.G.. Are some comparative nutrition claims misleading? The role of nutrition knowledge, ad-claim type, and disclosure conditions. J. Advertising, 29,29 $43,2000$.

24. Moorman C.: The effects of stimulus and consumer characteristics on the utilization of nutrition information. J. Consumer Res., 17, 362-374, 1990.

25. Petty R.E., Cacioppo J.T.: Communication and Persuasion: Central and Peripheral Routes to Attitude Change. New York, Sringer-Verlag, 1986.

26. Jacobson M., Corcoran L.: Snack attack: olestra. Nutrition Action Healthletter, (March), 9-11, 1998. 